\title{
Evaluation of automated breast volume scanner for breast conservation surgery in ductal carcinoma in situ
}

\author{
ANQIAN HUANG ${ }^{1}$, LUOXI ZHU ${ }^{1}$, YANJUAN TAN ${ }^{1}$, JIAN LIU ${ }^{2}$, \\ JINGJING XIANG ${ }^{3}$, QINGQING ZHU ${ }^{1}$ and LINGYUN BAO ${ }^{1}$ \\ Departments of ${ }^{1}$ Ultrasound, ${ }^{2}$ Breast Surgery and ${ }^{3}$ Pathology, The First People's Hospital of Hangzhou, \\ Affiliated Hangzhou Hospital of Nanjing Medical University, Hangzhou, Zhejiang 310006, P.R. China
}

Received May 5, 2016; Accepted July 22, 2016

DOI: $10.3892 / \mathrm{ol} .2016 .4924$

\begin{abstract}
The present is a retrospective study examining the use of automated breast volume scanner (ABVS) for guiding breast conservation surgery in ductal carcinoma in situ (DCIS). A total of 142 patients with pathologically confirmed DCIS were initially included in the study. The patients underwent preoperative examination by conventional ultrasound and by ABVS. The BI-RADS category system was used to identify benign and malignant lesions, after which breast conservation surgery was performed, and the therapeutic effects were compared. DCIS lesions were found in each quadrant of the breasts. Typical symptoms included: Duct ectasia and filling in 23 cases, mass (mainly solid, occasionally cystic, with or without calcification) in 38 cases, hypoechoic area (with or without calcification) in 33 cases, calcifications (simple) in 23 cases, and architectural distortion in 17 cases. In addition, 110 cases $(82.1 \%)$ were detected as grade $\geq 4$ according to the BI-RADS category, and 92 cases $(68.7 \%)$ were considered malignant lesions following conventional ultrasound scanning. The detection rate of ABVS was significantly higher than that of conventional ultrasound $\left(\chi^{2}=268.000, \mathrm{P}<0.001\right)$. The average tumor diameter was $2.5 \pm 0.8 \mathrm{~cm}$ using ABVS and $2.0 \pm 0.9 \mathrm{~cm}$ using conventional ultrasound (the former being significantly higher than the latter; $\mathrm{t}=6.325, \mathrm{P}=0.034$ ). Eight patients $(5.6 \%)$ had recurrences of the cancer, and the tumor diameter in the 8 patients was significantly larger using ABVS as compared to conventional ultrasound. In the diagnosis of DCIS, ABVS was superior to conventional ultrasound scanner in guiding breast conservation surgery and predicting recurrence. However, large-scale studies are required for confirmation of the findings.
\end{abstract}

Correspondence to: Dr Lingyun Bao, Department of Ultrasound, The First People's Hospital of Hangzhou, Affiliated Hangzhou Hospital of Nanjing Medical University, 261 Huansha Road, Hangzhou, Zhejiang 310006, P.R. China

E-mail: bao_lingyun1@163.com

Key words: automated, ultrasound, ductal carcinoma in situ, mammography, breast conservation surgery

\section{Introduction}

Ductal carcinoma in situ (DCIS) is a type of non-invasive cancer confined to the breast ductal system, without involvement of the basement membrane or the surrounding interstitium $(1,2)$. It is characterized by significant epithelial hyperplasia and presents characteristic mild to severe cellular atypism, which increases its risk of development into an invasive breast cancer (1). Therefore, the early detection and treatment are not only very effective but also imperative.

Newly identified DCIS cases account for $7.8-18.8 \%$ of breast cancer patients in China annually (2). Clinically, mammography X-ray and color ultrasound are the most commonly used diagnostic techniques. However, each method has important limitations: X-ray is only sensitive to calcification; and conventional ultrasound can be useful in estimating tumor size, echogenicity, morphology, perfusion, and other characteristics (3). Nevertheless, the results are highly variable and quantitative analyses cannot be conducted. An automated breast volume scanner (ABVS) can obtain cross-sectional, vertical, coronal and any other plane images, reflecting tumor features more clearly (4). Additionally, computer automatic orientation and calculations can make the results more objective and operator-independent (4). Furthermore, the breast imaging reporting and data system (BI-RADS) may be used to classified the benign and malignant tumors to guide the surgical treatment (5).

The aim of the study was to assess use of the ABVS and analyze the effects and prognosis of breast conservation surgery for DCIS to provide a reference for clinical treatment. The results showed that in the diagnosis of DCIS, ABVS is superior to conventional ultrasound scanner in guiding breast conservation surgery and predicting recurrence.

\section{Subjects and methods}

Subject information. A total of 142 DCIS female patients with breast lesions initially presented to The First People's Hospital of Hangzhou (Hangzhou, China) between January, 2011 and January, 2014 were selected for participation in the study. Eight patients did not present any abnormalities following examinations via mammography, MRI and fiberoptic ductoscopy, and were excluded. The remaining 134 patients that were enrolled 

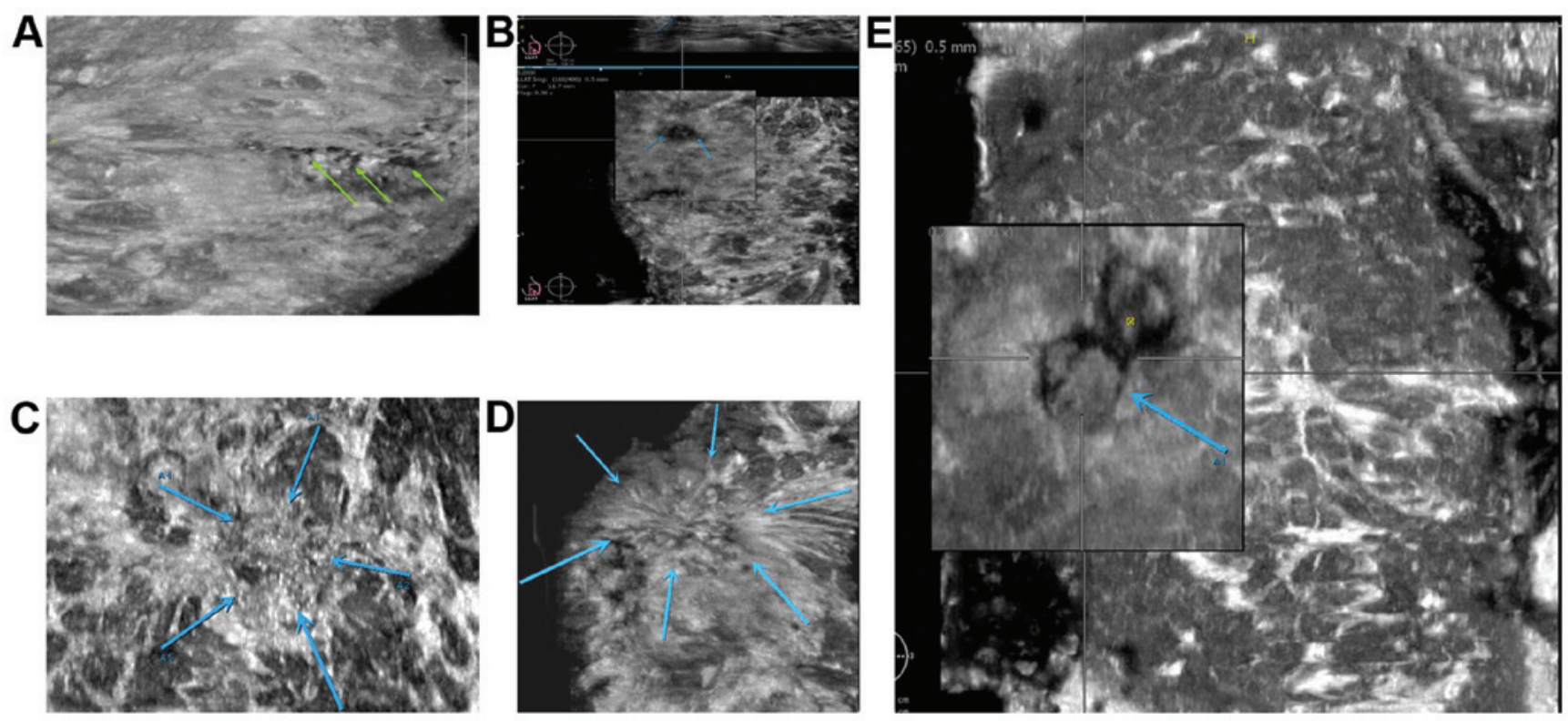

Figure 1. ABVS coronal plane image characteristics. (A) Duct ectasia and filling, (B) mass, (C) microcalcification, (D) architectural distortion, and (E) complex mass.

had surgical pathology results. The remaining 134 patients had had a single lesion and presented clinical features including plasmatic or serous nipple discharge, breast mass and nipple Paget disease. These patients underwent mammography X-ray, color ultrasound and ABVS, and they all had breast conservation surgery indications without contraindications. The same surgical and nursing teams in The First People's Hospital of Hangzhou performed all the surgeries in accordance with standard operation procedures.

The ethics committee of The First People's Hospital of Hangzhou approved the study. Patients and their relatives provided written informed consent.

ABVS examination method. The Acuson S2000 ABVS system (Seattle, WA, USA), a 14L5BV high frequency linear array probe, and $11 \mathrm{MHz}$ of center frequency were used to complete conventional three-dimensional scans of each side of each breast including the middle, inside and lateral positions, and the scanning orientation was increased when the breast was large. After scanning, volume data were transferred to the ABVS workstation to obtain plane basic images, and perform three-dimensional reconstructions with basic images of the whole breast including the vertical and coronal plane. Additionally, 18L6HD high frequency linear array probes made additional scannings for areas of interest to measure color Doppler parameters. The independent workstation of the ABVS system analyzed previously obtained DICOM data, measured the sizes of three lesion diameters of the largest plane of the three, and determined the shape, edge, internal echogenicity, and association with surrounding tissues rigorously. Image data were observed and analyzed independently by two experienced doctors. Assessment indexes for final classifications were based on the BI-RADS fifth version (ultrasound) revised by the American College of Radiology in 2013 (6), and suspected lesions were recorded, evaluated and compared uniformly and then divided into grade 0-6 according to the degree of danger, where grade $\geq 4$ were considered malignant tumors.

Clinical observations. Patients underwent a 3-year follow-up, where ABVS image characteristics were analyzed, and tumor diameter, BI-RADS grading and tumor recurrence were identified and recorded.

Statistical analysis. SPSS 19.0 software (SPSS, Inc., Chicago, IL, USA) was used for recording data and statistical analysis. Quantified data were presented as mean \pm standard deviation, and inter-group comparisons were carried out using the t-test. Qualitative data were presented as cases or percentages (\%), and inter-group comparisons were performed using the $\chi^{2}$ test. $\mathrm{P}<0.05$ was statistically significant.

\section{Results}

DCIS detection using BI-RADS category. In the cases studied, DCIS was found in all of the breast quadrants. Typical manifestations included duct ectasia and filling in 23 cases, a mass in 38 cases, calcification in 33 cases, architectural distortion in 23 cases, and solid complex masses in 17 cases. According to the BI-RADS classification, there were 24 probably benign grade $\leq 3$ cases (17.9\%); 54 probably malignant lesions needing biopsy (40.2\%): 9 grade 4A cases, 20 grade 4B cases, and 25 grade $4 \mathrm{C}$ cases; 26 highly suspected grade 5 malignant lesions requiring surgical resection $(19.3 \%)$; and 30 grade 6 malignant lesions (22.3\%). A total of 110 malignant cases were found using the ABVS system, and the detection rate was $82.1 \%$. By contrast, 92 cases $(68.7 \%)$ were considered malignant lesions using conventional ultrasound, the difference being statistically significant $\left(\chi^{2}=268.000 ; \mathrm{P}<0.001\right)$ (Table I).

Tumor diameters. ABVS showed the tumor diameter to range from 0.6 to $9.6 \mathrm{~cm}(4.5 \pm 0.8 \mathrm{~cm}$ on average), while conventional 
Table I. DCIS detection and tumor diameters.

\begin{tabular}{lcc}
\hline Parameters & $\begin{array}{c}\text { Detection rate } \\
\text { No. }(\%)\end{array}$ & $\begin{array}{c}\text { Tumor } \\
\text { diameter }(\mathrm{cm})\end{array}$ \\
\hline BI-RADS category & $110(82.1)$ & $4.5 \pm 0.8$ \\
Ultrasonography & $92(68.7)$ & $2.0 \pm 0.9$ \\
t-test $/ \chi^{2}$ test & 268.000 & 6.325 \\
P-value & $<0.001$ & 0.034 \\
\hline
\end{tabular}

ultrasound showed the diameters to range from 0.8 to $4.2 \mathrm{~cm}$ (2.0 \pm 0.9 on average). The tumor diameter using ABVS was significantly larger than that measured using conventional ultrasound, $(\mathrm{t}=6.325, \mathrm{P}=0.034)$ (Table I).

Eight cases (5.6\%) of recurrence of DCIS, and their tumor diameters were the largest by ABVS. However, their diameters were not larger when examined using conventional ultrasound (Fig. 1).

\section{Discussion}

On ABVS ultrasonography, duct ectasia and filling reflect local duct expansions, and dilating ducts are evident with or without a solid hyperechoic mass. In particular, on the coronal plane, ducts form 'tree branch' structures, and tufted calcified plaques can be detected in the vicinity. This provides clear signs for diagnosing DCIS (7). Common reasons for mistreatment in DCIS include instances where no clear malignant characteristics can be found, when there are intact edges, mean echogenicities, undetected calcifications, inconspicuous blood signals in manual scanning, and BI-RADS grades $\leq 3$ (8). Grit-like calcifications are scattering hyper-echoic calcification distributions, low glandular tissue echogenicity, and increased local blood flow signals (9). Architectural distortion manifests as local gland thickening and echo change with a range of 2.0-10.0 cm, calcification plaques, and different blood flow signals (10). Complex masses manifest as mixed cystic and solid echogenic masses with unclear boundaries between the thick wall and solid part, and present rich blood flow signals in the solid portions via manual scanning (11).

The ABVS breast coronal images obtained based on three-dimensional reconstruction can automatically measure the distance between the lesion, skin and nipple, and position the mass clearly within the breast boundaries, providing accurate location information regarding the mass (12). The maximum tumor diameter, one of the two biological indexes to predict breast cancer prognosis, has a negative correlation with the survival rate, provides evidence for tumor $\mathrm{T}$ phasing and affects the decision for the need of adjuvant chemotherapy, and is therefore an important index for preoperative evaluation before breast conservation surgery (13). Conventional ultrasound obtains the maximum value mainly through analysis of tumor cross-section and longitudinal section morphology. However, distortions of shape, position and brightness on ultrasonography may cause errors in its measurement (14). On the other hand, the ABVS coronal image conforms to the surgical vision, thus, it can better reflect edge features of the lesion and more easily identify the invasive range of cancers (15). The visual field width of the conventional ultrasound of 3-4 cm, limits to some extent the measurements of breast tumors with larger ranges, particularly DCIS in the form of continuous three-dimensional growths along a duct (16). The visual field width of ABVS is $15.4 \mathrm{~cm}$, which means it is able to avoid errors in measurement data caused by image mosaicisms (17). Notably, previous findings showed that, the size of breast tumor measured by ABVS is $98 \%$ accurate with a length deviation of $<2 \mathrm{~cm}$, supporting the hypothesis that ABVS can be used for planning breast conservation surgery (18).

ABVS has the following disadvantages: i) There are some patients who cannot have their breasts compressed or have poor scanning results, such as in cases of advanced breast cancer with diabrotic skin surface. ii) Cross sectional images, useful in the identification of superficial masses that are prone to slipping cannot be made. iii) Since ABVS provides two-dimensional grey-scale images, color Doppler, spectral Doppler and elasticity scanners are required to provide more complete information. iv) ABVS requires a large computer and a long period of time to process.

In summary, the detection rate of malignant tumors was higher than that of conventional ultrasound, and the tumor diameter detected using ABVS was also more accurate. Therefore, ABVS is superior to conventional ultrasound in diagnosing DCIS, guiding breast conservation surgery and predicting recurrence. The sensitivity, specificity, and accuracy of ABVS for evaluating suspicious breast lesions should be analyzed in a random clinical control trial with larger samples in order to provide the best reference for guiding surgery and improve prognosis.

\section{Acknowledgements}

The study was supported by the Science and Technology Program of Hangzhou (grant no. 20150633B18).

\section{References}

1. Yang WT and Zhu XZ: The introduction of 2012 WHO classification of tumours of the breast. Zhonghua Bing Li Xue Za Zhi 42: 78-80, 2013 (In Chinese).

2. Si W, Li Y, Han Y, Zhang F, Wang Y, Li Y, Linghu RX, Zhang X and Yang J: epidemiological and clinicopathological trends of breast cancer in Chinese patients during 1993 to 2013: A retrospective study. Medicine (Baltimore) 94: e820, 2015.

3. Bok SK, Jeon Y and Hwang PS: Ultrasonographic evaluation of the effects of progressive resistive exercise in breast cancer-related lymphedema. Lymphat Res Biol 14: 18-24, 2016.

4. Wojcinski S, Farrokh A, Hille U, Wiskirchen J, Gyapong S Soliman AA, Degenhardt F and Hillemanns P: The automated breast volume scanner (ABVS): Initial experiences in lesion detection compared with conventional handheld B-mode ultrasound: A pilot study of 50 cases. Int J Womens Health 3: 337-346, 2011.

5. Shen WC, Chang RF and Moon WK: Computer aided classifications system for breast ultrasound based on Breast Imaging Reporting and Data System (BI-RADS). Ultrasound Med Biol 33: 1688-1698, 2007.

6. Wöhrle NK, Hellerhoff K, Notohamiprodjo M, Reiser MF and Clevert DA: Automated breast volume scanner (ABVS): A new approach for breast imaging. Radiologe 50: 973-981, 2010 (In German).

7. Tozaki M, Isobe S, Yamaguchi M, Ogawa Y, Kohara M, Joo C and Fukuma E: Optimal scanning technique to cover the whole breast using an automated breast volume scanner. Jpn J Radiol 28: 325-328, 2010. 
8. Shin HJ, Kim HH, Cha JH, Park JH, Lee KE and Kim JH: Automated ultrasound of the breast for diagnosis: Interobserver agreement on lesion detection and characterization. AJR Am J Roentgenol 197: 747-754, 2011.

9. Zhang Q, Hu B, Hu B and Li WB: Detection of breast lesions using an automated breast volume scanner system. J Int Med Res 40: 300-306, 2012.

10. Isobe S, Tozaki M, Yamaguchi M, Ogawa Y,Homma K, Satomi R, Saito M, Joo C and Fukuma E: Detectability of breast lesions under the nipple using an automated breast volume scanner: Comparison with handheld ultrasonography. Jpn J Radiol 29 361-365, 2011

11. Li N, Jiang YX, Zhu QL, Zhang J, Dai Q, Liu H, Yang Q, Wang HY, Lai XJ and Sun Q: Accuracy of an automated breast volume ultrasound system for assessment of the pre-operative extent of pure ductal carcinoma in situ: Comparison with a conventional handheld ultrasound examination. Ultrasound Med Biol 39: 2255-2263, 2013.

12. Kim YW, Kim SK, Youn HJ, Choi EJ and Jung SH: The clinical utility of automated breast volume scanner: A pilot study of 139 cases. J Breast Cancer 16: 329-334, 2013.

13. Golatta M, Franz D, Harcos A, Junkermann H, Rauch G, Scharf A, Schuetz F, Sohn C and Heil J: Interobserver reliability of automated breast volume scanner (ABVS) interpretation and agreement of ABVS findings with hand held breast ultrasound (HHUS), mammography and pathology results. Eur J Radiol 82: e332-e336, 2013
14. Garcia-Uribe A, Erpelding TN, Krumholz A, Ke H, Maslov K, Appleton C, Margenthaler JA and Wang LV: Dual-modality photoacoustic and ultrasound imaging system for noninvasive sentinel lymph node detection in patients with breast cancer. Sci Rep 5: 15748, 2015.

15. Chen L, Chen Y, Diao XH, Fang L, Pang Y, Cheng AQ, Li WP and Wang Y: Comparative study of automated breast 3-D ultrasound and handheld B-mode ultrasound for differentiation of benign and malignant breast masses. Ultrasound Med Biol 39: 1735-1742, 2013.

16. Hao SY, Ou B, Li LJ, Peng YL, Wang Y, Liu LS, Xiao Y, Liu SJ, Wu CJ, Jiang YX, et al: Could ultrasonic elastography help the diagnosis of breast cancer with the usage of sonographic BI-RADS classification? Eur J Radiol 84: 2492-2500, 2015

17. Giuliano V and Giuliano C: Improved breast cancer detection in asymptomatic women using 3D-automated breast ultrasound in mammographically dense breasts. Clin Imaging 37: 480-486, 2013.

18. Tozaki M and Fukuma E: Accuracy of determining preoperative cancer extent measured by automated breast ultrasonography. Jpn J Radiol 28: 771-773, 2010. 